\title{
Approximate 3D Body-Wave Synthetics for Tomographic Models
}

\author{
by Don V. Helmberger and Sidao Ni
}

\begin{abstract}
We present a new method of generating analytical synthetics for tomographic-style models. These models are perturbations to a 1D layered model involving changes in block velocities producing 3D images. The procedure is broken into three steps: (1) construction of ray paths for the reference 1D layered model, (2) generation of perturbed paths and the construction of 2D synthetics in the plane containing the source and receiver, and (3) addition of out-of-plane contributions (2D) from virtual receivers weighted by diffraction operators. In step 1, the ray paths reflecting from the various interfaces are established with ray parameter $\left(p_{\mathrm{o}}\right)$ and travel time $\left(t_{\mathrm{o}}\right)$. Next, these values are corrected after adding the velocity perturbations where ray segments in faster blocks grow relative to slower blocks. This new set of ray parameters can be used to generate 2D Cagniard-deHoop synthetics or WKM synthetics. Contributions from virtual receivers at neighboring azimuths are added by convolving with diffraction operators that are defined by the source duration and travel time to the 3D structure. We suggest a particularly simple approximation based on four virtual receivers which produces synthetics in agreement with $3 \mathrm{D}$ numerical synthetics.
\end{abstract}

\section{Introduction}

The largest low-velocity structures in the lower mantle occur beneath southern Africa and the mid-Pacific. These low shear-wave velocity structures are correlated with hotspots (Williams et al., 1998), suggesting that the decrease in velocity is associated with an increase in temperature. The positive geoid anomaly and high topography over Africa (Hager et al., 1985), and the broad-scale Cenozoic uplift (Gurnis et al., 2000), are all consistent with warm, rising mantle beneath Africa. However, recent tomographic imaging of the whole mantle suggests that bulk sound velocity $\left(V_{\mathrm{c}}\right)$ and shear velocity $\left(V_{\mathrm{s}}\right)$ are negatively correlated within the African and Pacific anomalies (Masters et al., 2000), suggesting that the anomalies are chemical in origin, not thermal. The chemical hypothesis is supported by an inversion of normal modes (Ishii and Tromp, 1999), which indicates that the density of the lower mantle may increase within the two low $V_{\mathrm{s}}$ anomalies. However, if high-density chemical anomalies are embedded within thermal upwellings, we would predict that there should be sharp jumps in seismic velocity, either radially or laterally as found in dynamic models (Tackley, 2000). Thus, the sharpness of structures becomes a key issue in distinguishing between temperatures versus chemistry as the proper explanation of these anomalous structures.

A particularly effective means of determining lower mantle structure is through the study of differential travel times between $\left(S-S_{\mathrm{c}} S\right)$ and (SKS-S) (Ni and Helmberger, 2003a). Anomalous patterns in South African data required enhancing and sharpening of existing tomographic models to fit such data resulting in a ridge-like structure extending upward from the core-mantle-boundary (CMB) with a 3\% drop in shear velocity (see Fig. 1). Note that the phase SKS is nearly parallel to the eastern boundary, which predicts about a 6-s decrease in travel times between $96^{\circ}$ and $99^{\circ}$ (Fig. 1e). This jump occurs in less than a degree $(100 \mathrm{~km})$ or $50 \mathrm{~km}$ at the CMB in many data profiles ( $\mathrm{Ni}$ and Helmberger, 2003a). The SKS waveforms appear complex near this crossover, suggesting multipathing, which can be addressed with 2D WKM theory (Ni et al., 2002). We will briefly review this approach before introducing 3D effects in the next section, but first we will examine some observations sampling the ridge structure displayed in Figure $2 b$ for paths along the wall, at right angles to the paths in Figure $1 b$.

Observations recorded by the South African Array of deep Fiji events are particularly interesting as reported by Wen (2001). The $S_{\text {diff }}$ paths from one of the events (Western Pacific, 971222 at a depth of $180 \mathrm{~km}$ ) he used is displayed in Figure 2a. Note that some of the paths sample the northern dark-edge boundary. The waveform data are displayed in two formats, one in a conventional record section (Fig. 2b) and one as a "fan-shot" or as a function of azimuth. Normal observations should start at $t=0$. When plotted against azimuth, we see a systematic delay of data at $230^{\circ}$ (paths inside the structure) relative to paths near the edge, $246^{\circ}$. Wen (2001) modeled these waveforms very well by dividing 


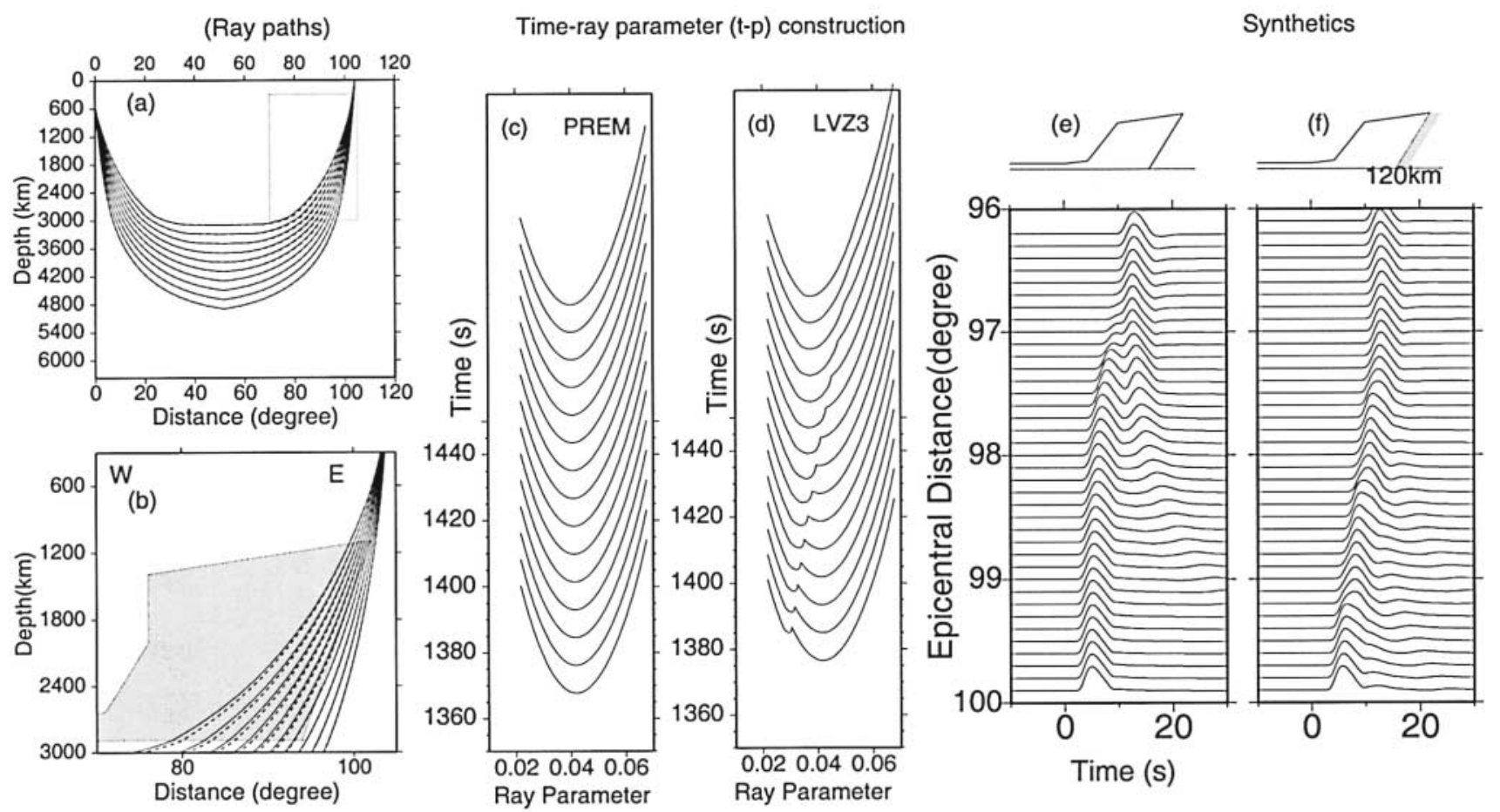

Figure 1. Composite display of steps taken in the generation of WKM synthetics. The column on the left displays ray paths with various $p_{\mathrm{i}}$ and $t_{\mathrm{i}}$ connecting the source to the receiver after reflecting from each interface. The panel (a) contains the $1 \mathrm{D}$ reference model (PREM) and panel (b) contains the model LVZ3 (gray zone contains 3\% drop in shear velocity). Panels (c) and (d) display the ( $p-t)$ curves for the SKS ray family as a function of distance, $96^{\circ}$ to $100^{\circ}$. The two local minimums near the mid ranges produce two discrete pulses at short periods as displayed on the right (e). The far right set of synthetics (f) is for a transitional model (120-km-wide boundary zone).

up the data into sectors (narrow windows of azimuth) with separate 2D models for each sector. Although some of these variations could well be $2 \mathrm{D}$, as he modeled it, some of it must be multipathing in azimuth (3D effects) as demonstrated in this study.

\section{Brief Review of Ray Theory Methods}

One of the most useful methods for generating 1D synthetics is called the WKBJ method developed by Chapman (1976). Results produced by this method are compared with reflectivity synthetics in Chapman and Orcutt (1985), where the various methods of computing synthetics are discussed in detail. Similar results involving generalized ray solutions are given in Burdick and Orcutt (1979) since these solutions have been well developed. We will start with the latter method where the wave field can be approximated by

$$
V(r, z, t)=\frac{d}{d t}[\dot{D}(t) * \phi(r, z, t)]
$$

where $V$ is the displacement for a flattened earth with $r$ the horizontal coordinate, $z$ the depth (Gilbert and Helmberger, 1972; Helmberger, 1973), where

$$
\phi(r, z, t)=\sqrt{\frac{2}{r}} \frac{1}{\pi}\left[\frac{1}{\sqrt{t}} * \psi(t)\right],
$$

with

$$
\psi=\operatorname{Im} \sum_{i=1}^{n} S R_{j}(p) \frac{\sqrt{p}}{\eta_{1}} R(p) \Pi_{i}(p)\left(\frac{d p_{i}}{d t_{i}}\right)
$$

and where $p=$ ray parameter and $S R_{i}(p)$ is the vertical radiation pattern; $\eta_{i}=\left(1 / \beta_{i}^{2}-p^{2}\right)^{1 / 2} ; \beta_{i}=$ shear velocity $(\mathrm{km} / \mathrm{s}) ; R(p)=$ receiver function; and $\dot{D}(t)$ is the far-field dislocation time history. The generalized rays are summed over $i$ with $\Pi_{i}(p)$ containing the product of transmission coefficients and the reflection coefficient at the reflected interface $(i)$. Equation (3) is essentially the line-source solution, whereas the convolution and distance correction of equation (2) transform it into a point-source solution. It is relatively easy to add shear dislocations to this formalism in the generation of synthetics (i.e., Helmberger, 1983). form:

The travel time associated with each ray has a simple

$$
t_{i}=p_{i} r+\sum T h_{k} \eta_{k}
$$




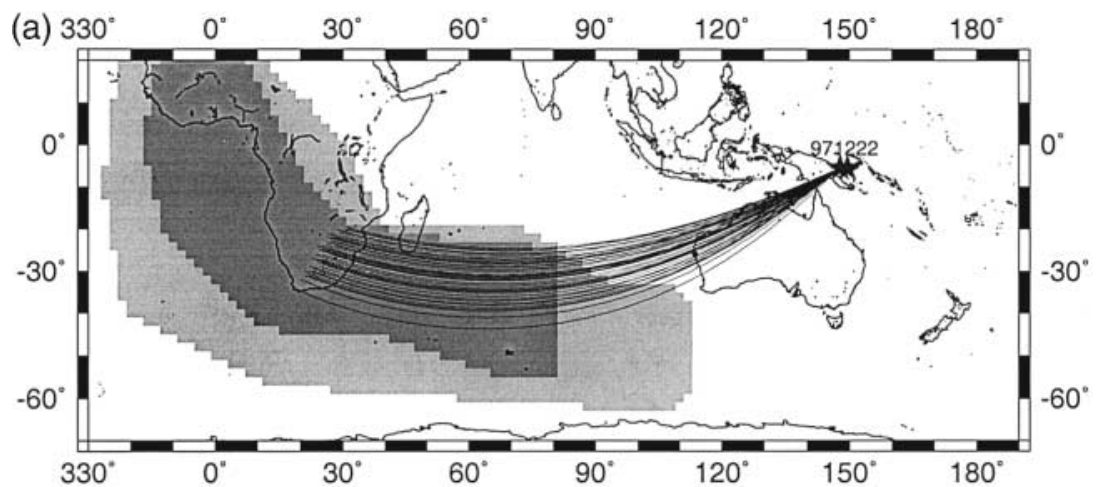

(b)

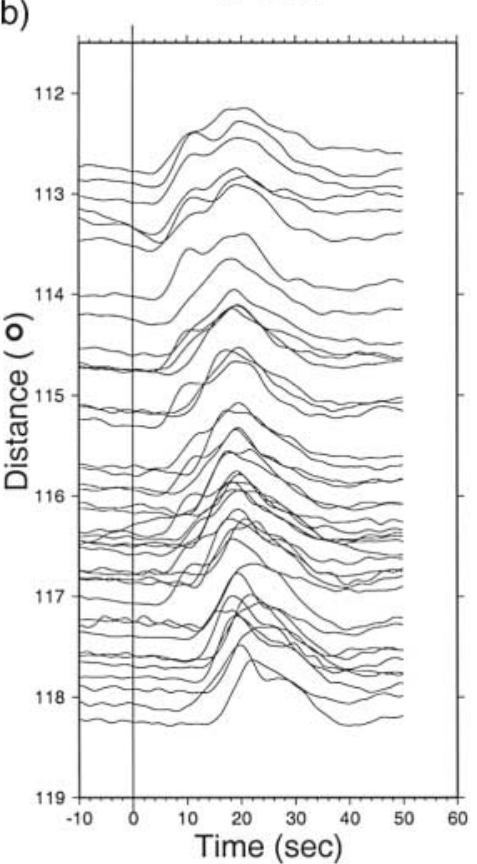

(c)

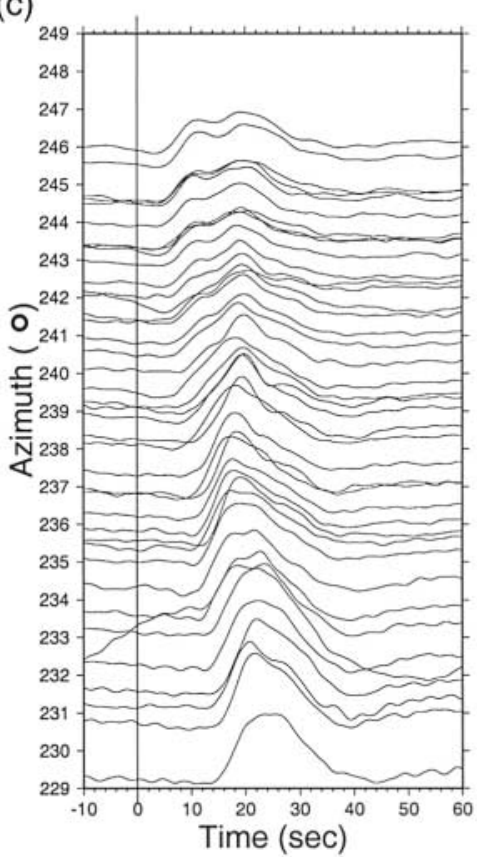

Figure 2. Panel (a) displays the ray paths encountering the 3D structure proposed by $\mathrm{Ni}$ et al. (2005). The light shading indicates the bottom $\mathrm{D}^{\prime \prime}$ structure and the dark shading indicates the uppermost structure, extending upward, $1200 \mathrm{~km}$ above the CMB. The entire structure has a uniform 3\% drop in shear velocity relative to PREM. The structure outside is Grand's tomographic model. Panels (b) and (c) display the $S_{\mathrm{d}}$ observations recorded by the array. Panel (b) displays observations recorded at the South African Array plotted in a record section, relative to PREM travel-time predictions. A plot of these data as a function of azimuth (c) reveals a strong case for azimuthal multipathing. where the summation is over the layers (thickness, $T h_{k}$ ) along the ray paths. The derivative $\left(d t_{i} / d p_{i}=0\right)$ at the stationary points where the rays satisfy Fermat's principle and obey Snell's law. This $p$ is labeled $p o$ and the corresponding time (to).

An example of such paths is displayed in Figure 1a where the phase SKS for the reference 1D model PREM is presented. The factor $d p_{i} / d t_{i}$ is the complex derivative of the ray parameter against time along the Cagniard-deHoop contours.

However, for simple core velocities, this method can be greatly simplified by examining

$$
\frac{\delta\left(p o_{i}-p o_{i-1}\right)}{\delta\left(t o_{i}-t o_{i-1}\right)}=\left(\frac{\delta p_{i}}{\delta t_{i}}\right)
$$

for various $i$ 's which has a square-root singularity at layer $i$ nearest the geometric ray stationary point (Chapman, 1976). The ( $p-t)$ curves in Figure 1c and d (PREM) display this feature as a function of epicentral distances, and allow equation (4) to be approximated by

$$
\Psi=S R_{j}(p) R(p)(\delta p / \delta t)\left(T_{\mathrm{SP}} \cdot T_{\mathrm{PS}}\right)
$$

where the first-order transmission coefficients for the CMB are retained. If one allows the velocities in equation (4) to vary (tomographic approximation), the $p$ - $t$ curves do not change appreciably. However, if one allows the ray parameter to be adjusted to obey Snell's law (Fig. 1b), the paths will flatten for segments with fast velocities and produce sharp features in the $(p-t)$ curves as for model LVZ3 displayed in Figure 1c and d (see Ni et al., 2000). Thus, we obtain two pulses with the most favorable geometry with the fast path producing the first pulse and the second pulse following the slow path. If we add a transition zone (second column) along the boundary, we no longer produce multipathing because of the smoothness of the $(p-t)$ curves (Ni et $a l ., 2002)$. These features can also be seen in 2D numerical 
synthetics generated for tomographic models (Ni et al., 2003a, 2003b). Note that the $\psi$ function is nearly the linesource solution except for the inclusion of the $(\sqrt{p})$ factor. This feature is exploited in developing 2D numerical simulation of earthquake (dislocation) sources (Helmberger and Vidale, 1988; Wen and Helmberger, 1998). To produce a point-source solution, we simply perform the operations in equation (2) which involves a convolution with $1 / \sqrt{t}$. This operation creates a step function-like response and is appropriate for models containing azimuthal uniformity. If the ray paths are traveling along a boundary (in or out of the paper in Fig. 1), this assumption fails. Thus, we will modify equations (2) and (3) to include effects of off-great-circle paths treated as diffractions.

\section{Diffraction Theory and Interfacing Wave Fields}

Interfacing numerical and integral techniques allows flexibility in seismic modeling and is becoming a part of the standard tools discussed in textbooks. Some recent applications involve 2D deep-earth models (Wen and Helmberger, 1998), and mapping abrupt jumps in crustal thickness (Zhu and Helmberger, 1998). Although there are many possible modifications (Frazer and Sen, 1985), the approach followed here is commonly referred to as the KirchhoffHelmholtz integral equation (Born and Wolf, 1964), with $\phi_{p}$ the potential at position $r$.

$\phi_{p}(r, t)=\frac{1}{4 \pi} \int_{S}-\frac{1}{r}\left(\frac{\delta u}{\delta n}+\frac{u}{r} \frac{\delta r}{\delta n}+\frac{1}{\alpha} \frac{\delta r}{\delta n} \frac{\delta u}{\delta t}\right) d S$

where $u$ is the wave field (potential) on the surface $(S)$ retarded by $\tau=t-t_{p}$, with $t_{p}$ the travel time from each element to the receiver. A unit vector " $n$ " denotes the direction perpendicular to the local surface. The integration is over the surface $S$ with elements $d S$.

This integral is simply a formal description of Huygen's principle, which states that the disturbance at some later time $\left(t_{p}\right)$ can be obtained by summing secondary sources on a surface $(S)$. To demonstrate its use, we will consider a simple application in a situation where the answer is known. The model setup is displayed in the upper panel of Figure 3 involving deep ocean sediments with a source in the ocean and receiver below the water-mud interface. The generalized ray solution in equation (2) for element points along the interface $(r)$ are displayed on the left in Figure 3, and are a function of depth $(h)$ on the right. The lowermost pair of traces displays the comparison of a summation (640 elements) at a depth of $125 \mathrm{~m}$ with the analytical solution. Note that this smooth looking response was produced by summing spikelike pulses properly lagged and weighted by $(\partial r / \partial n)$, cosines from equation (7). The response becomes rough when adding fine-scale variation (Scott, 1985). As the depth grows, the head wave evolves into a step and the tunneled direct wave disappears. This allows idealized solutions (geometric

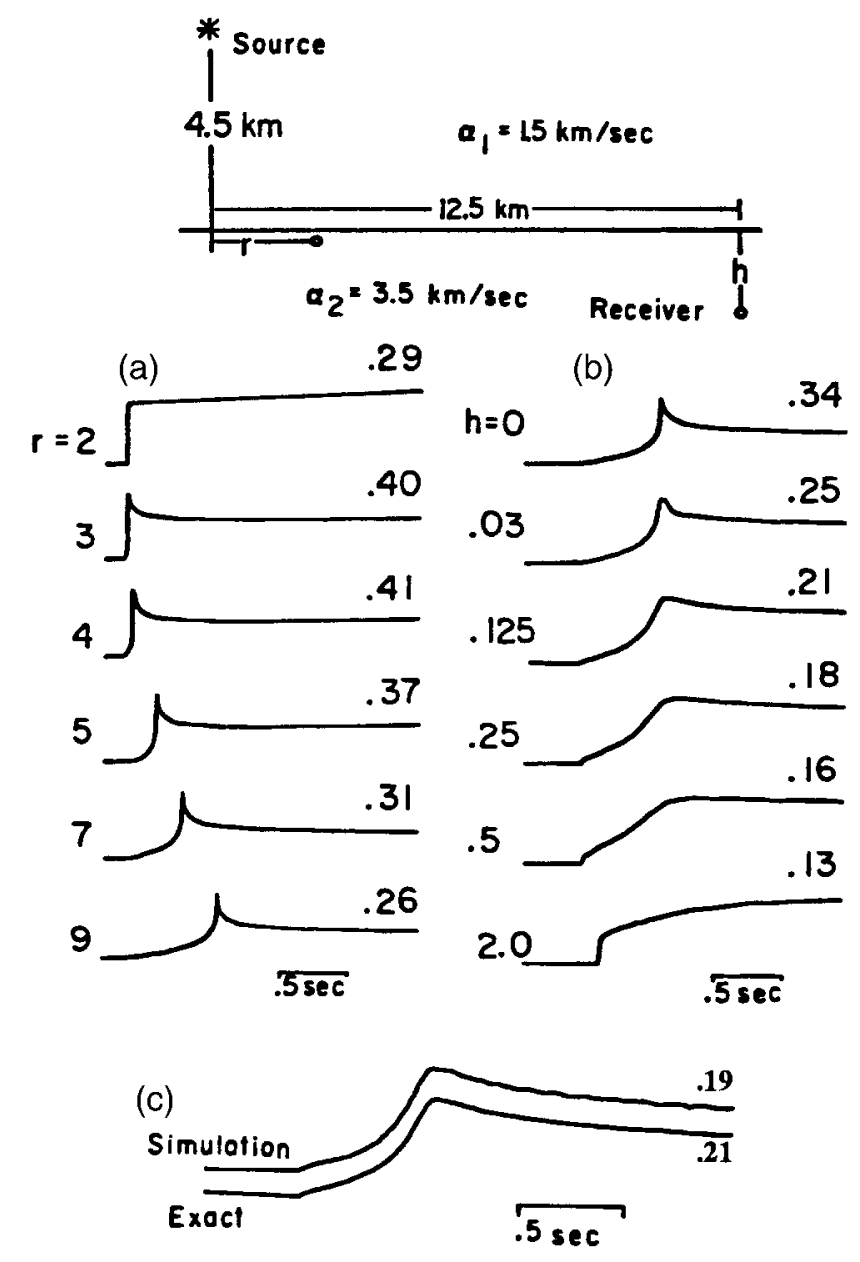

Figure 3. Panel (a) displays an example of Green's functions (in displacement) of the response just below the surface interface assuming a stepfunction source. The response is nearly that of the direct arrival at $r=2$. For larger $r$, the response develops a head wave. Panel (b) displays the CagniarddeHoop response as a function of depth situated at $r=6 \mathrm{~km}$. A comparison for $h=0.125 \mathrm{~km}$ between exact (Cagniard-deHoop) and simulation is given in panel (c).

rays) without the need of generating the full CagniarddeHoop Green's functions. An example of this type of solution is displayed in Figure 4 involving a step along an interface; see Scott and Helmberger (1985) for an assortment of structural shapes. The split of the responses for paths along the step corresponds to the classical result (half of response) and easily derived in both $2 \mathrm{D}$ and $3 \mathrm{D}$.

First, we rewrite equation (7) in Laplace transform space,

$$
\bar{\phi}_{p}(r, s)=\frac{1}{4 \pi} \int_{S}-\frac{1}{r}\left(\frac{\delta \bar{u}}{\delta n}+\frac{\bar{u}}{r} \frac{\delta r}{\delta n}+\frac{1}{\alpha} \frac{\delta r}{\delta n} s \bar{u}\right) d S
$$

with $s$ the transform variable. We assume a symmetric point source (Fig. 5) which lights up the interface 


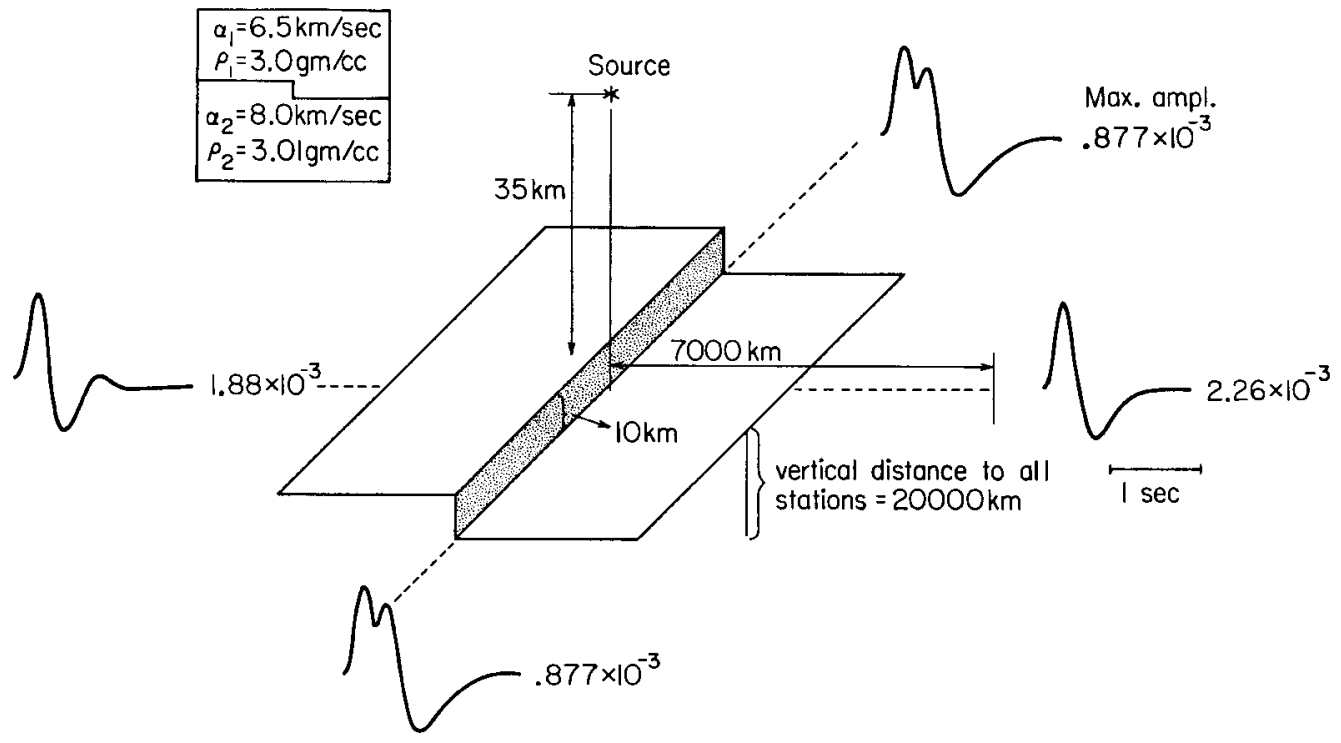

Figure 4. Generation of the transmitted response below a faulted-Moho with two pulses formed along the azimuth of the fault and larger single pulses at right-angle positions.

(a)

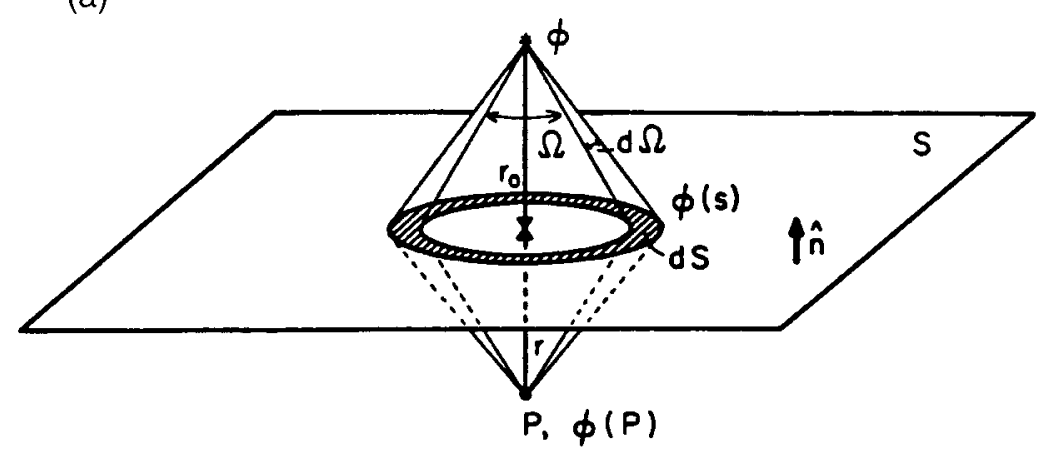

(b)

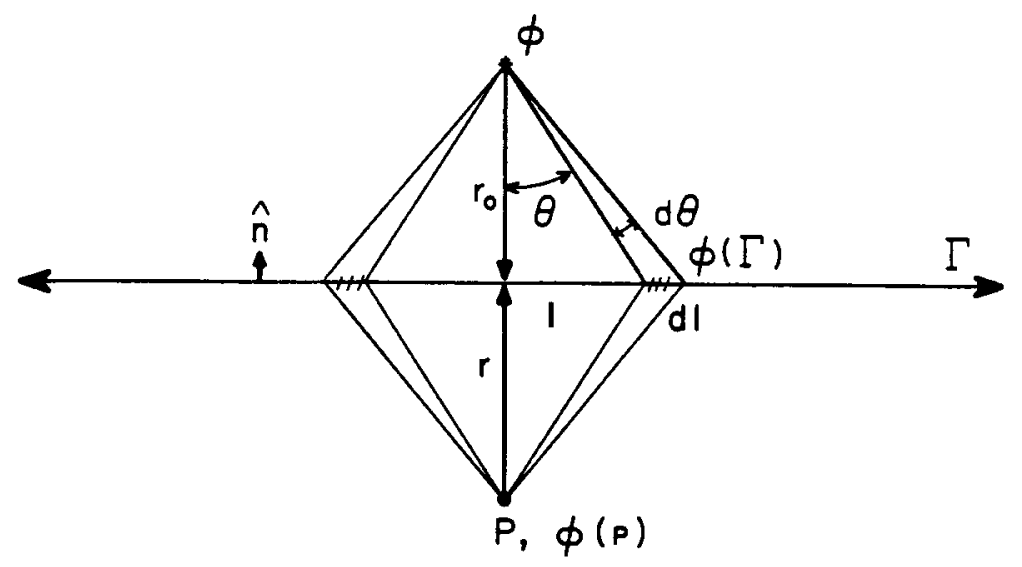

Figure 5. Geometry for 3D analytic evaluation of the Kirchhoff integral in (a). The interface surface is $S$, with solid angle $\Omega$ tracing out $d S$. The normal to the surface is $\hat{n}$. The source, at distance $r_{\mathrm{o}}$ from $S$, generates potential $\boldsymbol{\phi}$ which produces $\boldsymbol{\phi}(S)$ on the surface. Kirchhoff integration gives the result $\phi(P)$ at point $P$, at a distance $r$ from $S$. Geometry for analytic evaluation of the $2 \mathrm{D}$ Kirchhoff integral in the lower panel (b). The line interface is $\Gamma$, with angle $\theta$ tracing out $d l$. The normal to the contour is $\hat{n}$. The source, at distance $r_{\mathrm{o}}$ from $\Gamma$, generates potential $\phi$, which produces signal $\phi(\Gamma)$ on the surface. 2D Kirchhoff integration gives the result $\boldsymbol{\phi}(P)$ at point $P$, at a distance $r$ from $\Gamma$. 


$$
\bar{u}(s)=\frac{1}{r_{\mathrm{o}}} e^{-s r_{\mathrm{o}} / \alpha} \bar{f}(s)
$$

and assume $|r|=\left|r_{\mathrm{o}}\right|$ and $\delta r / \delta n=-\delta r_{\mathrm{o}} / \delta n$. We also suppose that near-field terms can be dropped or travel time is large compared with source duration. Note that

$$
d \Omega=\left(1 / r^{2}\right)\left(\frac{\delta r}{\delta n}\right) d S, \text { where } \Omega \text { is the solid angle. }
$$

Then

$$
\bar{\phi}_{p}(r)=\bar{f}(s) \frac{1}{2 \pi} \int_{\Omega} e^{-2 s r / \alpha}\left(\frac{s}{\alpha}\right) d \Omega
$$

Letting

$$
t=\frac{2 r}{\alpha} \text { and } d \Omega=\frac{d \Omega}{d t} d t
$$

we obtain

$$
\begin{aligned}
\phi_{p}(r)=\frac{d}{d t}\left[f(t) * \frac{1}{2 \pi} \frac{1}{\alpha} d \Omega / d t\right] \\
=\left[f(t) * \frac{1}{2 \pi} \frac{1}{\alpha} \frac{d^{2} \Omega}{d t^{2}}\right]
\end{aligned}
$$

where $\Omega(t)$ is the solid angle swept out over the surface (Hilterman, 1975).

$$
\Omega(t)=2 \pi\left(1-\frac{t_{\mathrm{o}}}{t}\right) H\left(t-t_{\mathrm{o}}\right)
$$

where $t_{0}$ is the first arrival (geometric). Note that $\Omega(t)=0$, $t=t_{0}$ and equal to $2 \pi$ as $t \rightarrow \infty$, and that

$$
\frac{d \Omega^{2}}{d t^{2}}=2 \pi \frac{t_{\mathrm{o}}}{t^{2}} \delta\left(t-t_{\mathrm{o}}\right)
$$

and

$$
\phi_{p}(r)=\frac{1}{R} f\left(t-t_{\mathrm{o}}\right), \text { since } t \alpha=R
$$

If the left plane is shifted up or down relative to the right plane, we get two pulses with a small timing offset as in Figure 4. Note that the amplitude is strongly controlled by the $(d \Omega / d t)$ which is always large for direct arrivals (stationary). Sharp changes in the interface or in the wave-field timing introduce complexity as discussed in Scott and Helmberger (1985).

A similar result can be derived from line-source theory as discussed by Stead and Helmberger (1988),

$$
\bar{\phi}_{\mathrm{L}}(r, s)=\bar{f}(s) \frac{1}{4} \int e^{-2 s r / \alpha} \frac{1}{r} \frac{\delta r}{\delta n} d l
$$

their equation 47. This integral is over the line with $d l$ the line element and $(\partial r / \partial n)$ the gradient direction. Now, from the geometry displayed in Figure 5, we can express

$$
d \theta=\frac{1}{r} \frac{\delta r}{\delta n} d l
$$

and

$$
\phi_{\mathrm{L}}(r, t)=f(t) * \frac{1}{2} \frac{d \theta}{d t},
$$

where

$$
\frac{d \theta}{d t}=2 \frac{t_{\mathrm{o}}}{t} \frac{H\left(t-t_{\mathrm{o}}\right)}{\left(t^{2}-t_{\mathrm{o}}^{2}\right)^{1 / 2}} .
$$

Note that if the right or left side of the interface were opaque, we would obtain only half the response. If the opaque region is extended, we lose the singularity and produce a shadow zone.

$$
\phi_{\mathrm{L}}(r, t)=f(t) * \frac{1}{2} \frac{H\left(t-t_{\mathrm{s}}\right)}{\left(t^{2}-t_{\mathrm{o}}^{2}\right)^{1 / 2}},
$$

since $H\left(t-t_{s}\right)$ does not turn on until $t_{s}$. Thus we have a simple way of weighting $2 \mathrm{D}$ diffractions as their distance away from a square-root singularity, which can be used to approximate the contributions from non-great-circle paths.

We construct a reference plane at a constant depth just above the 3D structure of interest. Note that we could compute the response lighting up this surface from below using the WKM code and then sum these secondary sources by performing a surface integral as discussed earlier in Figure 3. Moreover, interchanging the source and receiver produces the same seismograms and that the latter predicts multipathed $P$ waves from teleseismic distances such as in Zhu and Helmberger (1998). The construction of a reference plane for such a situation is displayed in Figure 6a where we construct a set of nonuniform elements specified by $\Delta X_{j}$ along a line of constant backazimuth, or at a right angle to the Great Circle. To define the $\Delta X_{j}$ spacing, we suppose that the distance from the plane to the receiver is $h$ and $X_{j}$ is the distance from 0 to $j$. Let $\Delta h+h$ be the path from $j$ to the receiver and assume $X \ll h$. Then,

$$
X_{j}=\sqrt{2 h \Delta h_{j}}=\sqrt{2 h \alpha \delta t_{j}}
$$

where $\alpha$ is the velocity and $\delta t_{j}=t_{j}-t_{o}$. If we compare $\delta t_{j}$ with the source duration $(T)$, we can predict some of the expected waveform complexity. In particular, if $\delta t_{j}<T / 2$, 

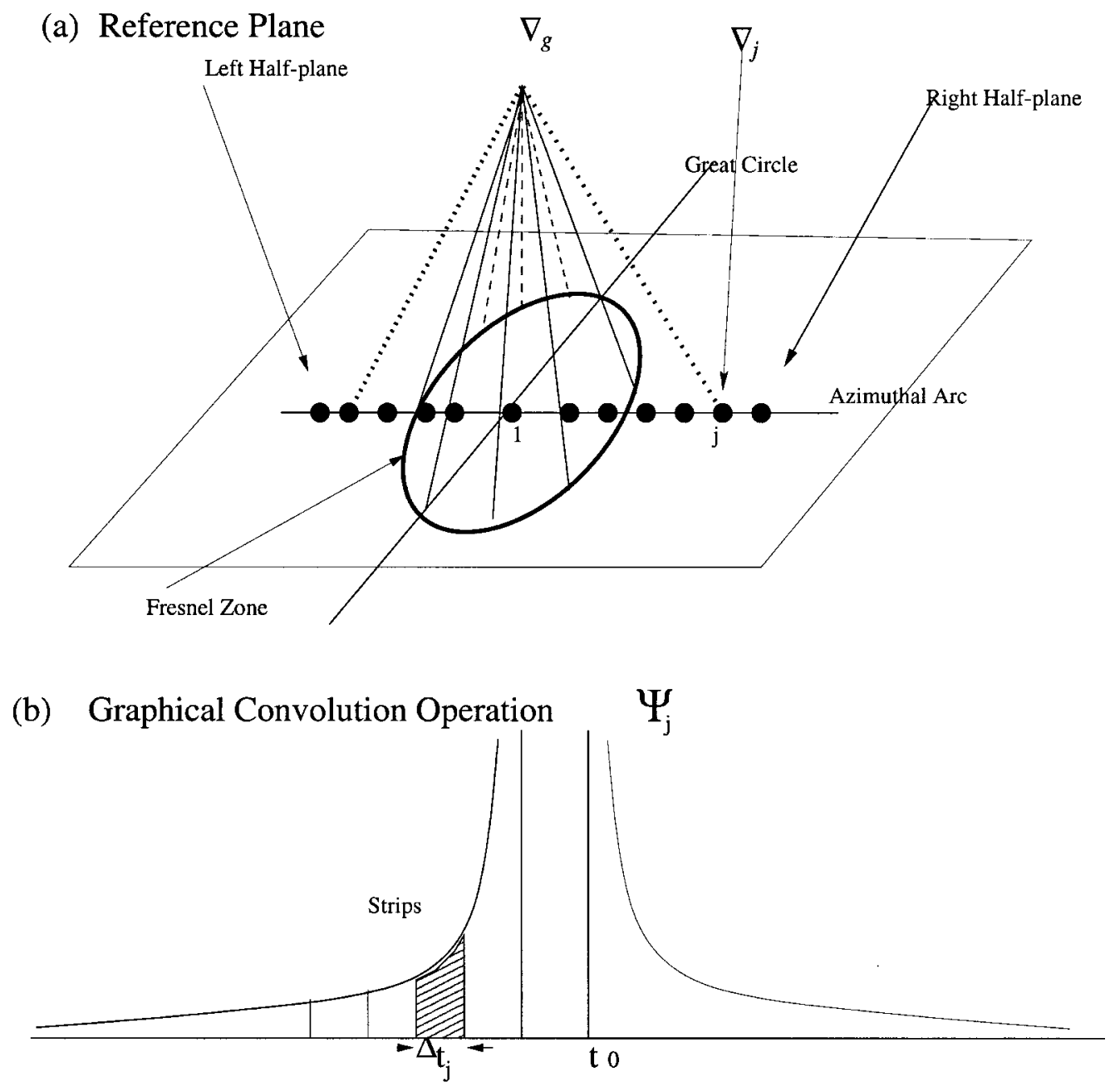

Figure 6. Construction of a reference plane (a) along the horizontal, essentially parallel to the Earth's surface. The ray path connecting the source with the receiver $\left(\Delta_{\mathrm{g}}\right)$ is arriving along the great circle line. A line along the azimuthal arc is included as well as a plot of the Fresnel zone. The position $\Delta_{j}$ is the location of a virtual receiver. The filled dots are the locations of spacial offsets from the piercing point (1) of the geometric ray specified by equal $\Delta_{t}$ delays. The lower panel (b) displays the graphical operation of the convolution of with a square-root singularity representing the $\psi_{j}(t)$ operator located at $t_{\mathrm{o}}$. Thus, the virtual receivers ( $j$ 's) nearest (1), the geometric ray, produce the sharpest and strongest contributions.

the pulses will simply average as well known (i.e., Červeny and Soares, 1992). Thus, one can define a Fresnel zone where differential arrivals are confined to be less than (T/2) and the Fresnel boundary becomes

$$
X=\sqrt{h \alpha T}
$$

or

$$
X=\sqrt{\lambda h} \text { with } \lambda \text { the wavelength. }
$$

Returning to our general formalism, we define the differential times

$$
\Delta t_{j} \equiv \delta t_{j+1}-\delta t_{j}
$$

and obtain

$$
\delta X_{j}=\sqrt{2 h \alpha \Delta t_{j}}
$$

We then compute the wave field $\Psi_{j}$ at the various virtual receivers $\left(X_{j}\right)$ and replace $[1 / \sqrt{r} * \psi(r, z, t)]$ in equation (2) with

$$
\frac{1}{2} \sum_{i=1}^{n}\left[\frac{H\left(t-t_{i}\right)}{\sqrt{t+\Delta t_{i}}} * \psi_{j}(t)-\frac{H\left(t-t_{i+1}\right)}{\sqrt{t+\Delta t_{i+1}}} * \psi_{j}(t)\right]
$$

where $t_{i}$ is the travel time from each sample to the receiver $\left(\nabla_{\mathrm{g}}\right)$ and $\Delta t_{i}=t_{i}-t_{\mathrm{o}}$, the difference in timing between the true geometric path and the diffracted path $(i)$. The $\psi_{j}(t)$ are 
computed at $\Delta X_{i}$ away from the geometric path. The $\psi_{j}$ values can be early or late caused by the (2D) structure, but they are all lagged by the $\Delta t_{i}$ caused by the extra travel path (corrected for being off azimuth) and systematically losing their strengths and high-frequency with separation. A graphical presentation of the convolution operator is displayed in Figure $6 \mathrm{~b}$. For the 2D case, all the $\psi_{j}=\psi$, the response computed at the true receiver and because $\psi$ behaves like a simple square-root singularity for simple situations (Chapman, 1976), we obtain a step-function turning on at $t_{\mathrm{o}}$ (i.e., Helmberger, 1983). But for the 3D case, we integrate in strips weighted relative to the geometric arrival and summing the responses from the right and left. If these two responses are offset in timing, such as in Figure 4, we would expect two pulses as displayed after applying equation (1). Thus, we can greatly simplify the planar grid summation as discussed in Figures 3 and 4. Still further approximations can be applied based on the Fresnel zone ideas as discussed in the next section.

\section{Application to the African Plume Structure}

In this section, we will apply the combination of WKM and diffraction (referred to as DWKM) to approximate synthetics for a 3D model. Thus we will attempt to predict some of the strong azimuthal effects discussed earlier in Figure 2. The edge and sharpness of this boundary were determined by mapping the jumps in SKS as observed on the African Array for a large number of earthquakes at all azimuths (Ni et al., 2003b). The first column in Figure 7 displays the 2D synthetics where only the in-plane motions are included. Note the jump in timing that occurs at the top edge of the figure when the ray paths first sample the slow plume structure. To include the out-of-plane contribution, we apply the

2D $3 \mathrm{D}(8 \mathrm{sec})$ $3 D(16 \mathrm{sec})$

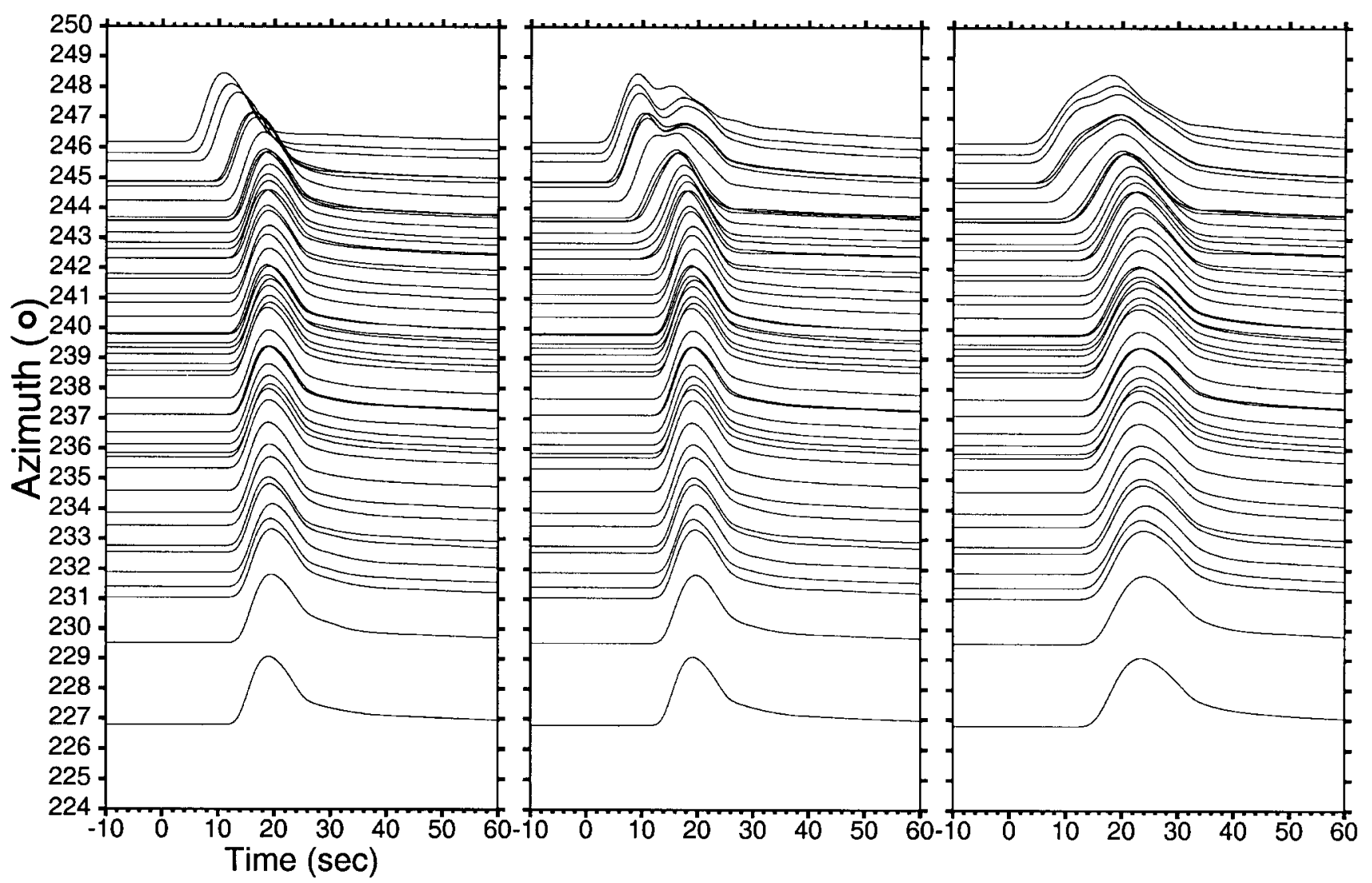

Figure 7. The column on the left displays 2D WKM synthetics $\left(S_{\text {diff }}\right)$ for a 3D model proposed by $\mathrm{Ni}$ and Helmberger (2003b). The traces at the top follow paths sampling a simple PREM earth except for a thin slow $\mathrm{D}^{\prime \prime}$ layer (3\%). The paths on the bottom (beyond $242^{\circ}$ ) sample the super plume, which are delayed about $15 \mathrm{~s}$. The middle column contains 3D DWKM synthetics where the response is simulated from four virtual sources, two on each side of the receiver at distances $134 \mathrm{~km}$ (lit zone) and 322 $\mathrm{km}$ (diffracted zone) away from the true position, assuming an 8-s duration. The column on the right is for a 16-s source duration with virtual positions $\pm 190 \mathrm{~km}$ and $456 \mathrm{~km}$, respectively. 
diffractions along an arc at constant epicentral distance as displayed in Figure 8.

We construct a reference plane at a constant depth just above the 3D structure of interest. We could compute the response lighting up this surface from below using the WKM code and then sum these secondary sources by performing a surface integral as discussed earlier. However, we simplify this procedure by replacing this integral with a line integral as displayed in Figure 8a and apply the weighting suggested by equation (16) in the preceding section.

However, this formalism can be made still simpler by adding some more approximations. Let the depth of the reference plane be $h$ and $\alpha$ be the average velocity along the path to the surface. Let $\Delta X$ be the horizontal distance associated with the diffracted paths (i.e., $\Delta_{\mathrm{g}}-\Delta_{\mathrm{f}}$ ) then

$$
\Delta X \approx \sqrt{2 \alpha \tau h}
$$

where $\tau$ is the differential time $\left(\Delta t_{i}\right)$ between the geometric

(a)

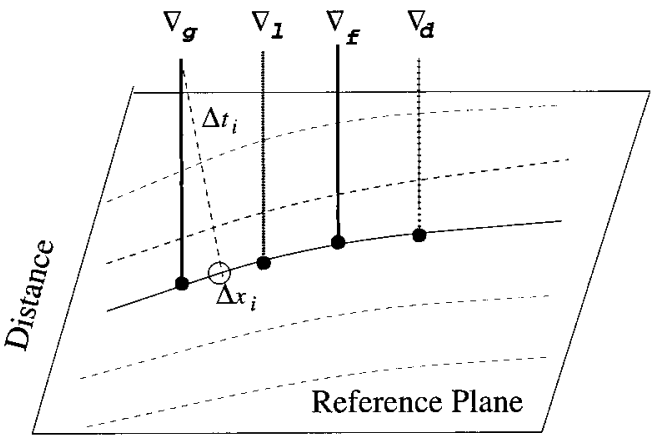

Azimuth

(b)

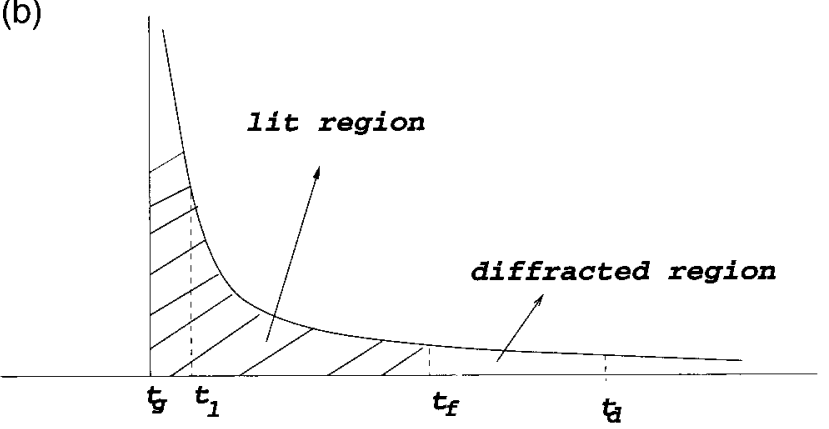

Figure 8. A surface of constant depth serving as a reference plane for interfacing Kirchhoff solutions is presented in panel (a). To shorten the calculation, we assume 2D geometry and integrate along the arc (heavy line) which corresponds to constant epicentral distance with variable azimuth. The other dotted lines correspond to other neighboring distances. Four locations are indicated corresponding to projections to the surface of the geometric arrival $\left(\nabla_{\mathrm{g}}\right)$, the Fresnel boundary $\left(\nabla_{1}\right)$ and two virtual stations $\nabla_{1}$ and $\nabla_{\mathrm{d}}$, representing the lit region and the diffracted region. The lower diagram (b) displays the time corresponding to the above four positions relative to the square-root weighting. time and the path arriving $\Delta x_{i}$ away, assuming $\tau \ll$ total travel time, as in the preceding section. We suggest that, instead of integrating along the arc displayed in Figure 8a, we approximate it based on $T, h$, and our level of model definition. Essentially, $\Delta x$ values must be larger than our tomographic block sizes to warrant consideration, which means that we can approximate the square-root singularity. We define four times; $t_{\mathrm{g}}$ the geometric time at the receiver, $t_{\mathrm{f}}=T / 2$, the Fresnel zone limit, $t_{1}=T / 8$, the time defining the average area under the square-root singularity between $t_{\mathrm{f}}$ and $t_{\mathrm{g}}$,

$$
t_{\mathrm{d}}=\frac{(\sqrt{2}+1)^{2}}{8} T \text {, }
$$

the time position defining the average area beyond $t_{\mathrm{f}}$. These times can be substituted into equation (17) to estimate

$$
\begin{aligned}
& X_{\mathrm{f}}=\sqrt{\alpha h T} \\
& X_{1}=\sqrt{\alpha h T / 4} \\
& X_{\mathrm{d}}=(\sqrt{2}+1) X_{1}
\end{aligned}
$$

If we assume the reference plane situated at a depth of 1500 $\mathrm{km}$, an average shear velocity of $6 \mathrm{~km} / \mathrm{s}$, and source duration of $12 \mathrm{~s}$, we obtain $X_{l}=164 \mathrm{~km}$ and $X_{d}=396 \mathrm{~km}$. The synthetic at $\Delta_{\mathrm{g}}$ is then estimated by convolving the four WKM responses at the virtual receivers with the two operators $O_{\mathrm{L}}$ and $O_{\mathrm{D}}$ for the right and left positions. These operations are defined by:

\section{Source Time Functions}

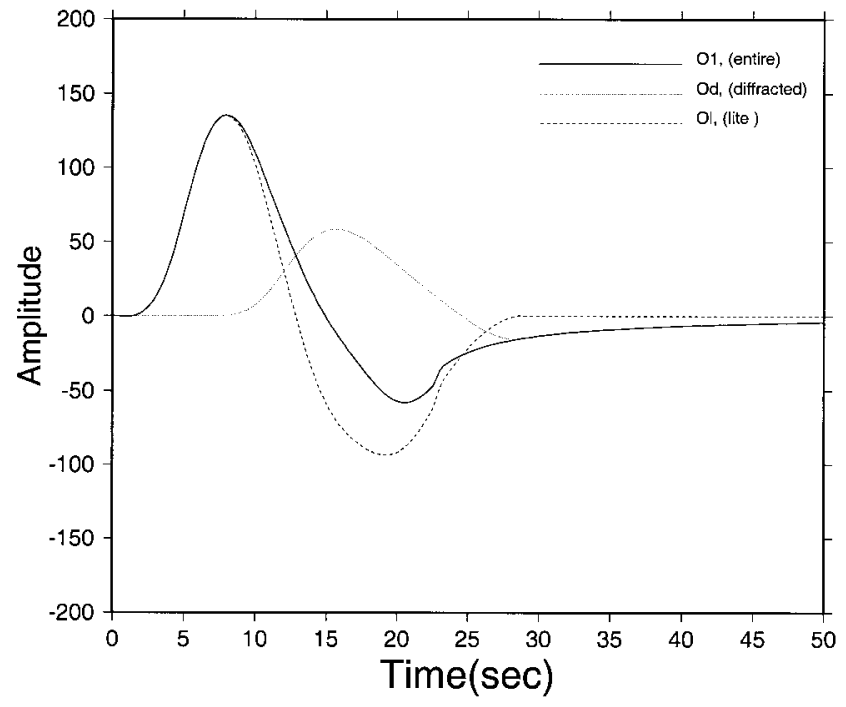

Figure 9. Operators corresponding to the lit region (light line), the diffracted region (dotted line), and 1D case (heavy line). These operators are convolved with WKM responses (2D) to construct the solution. 
DWKM

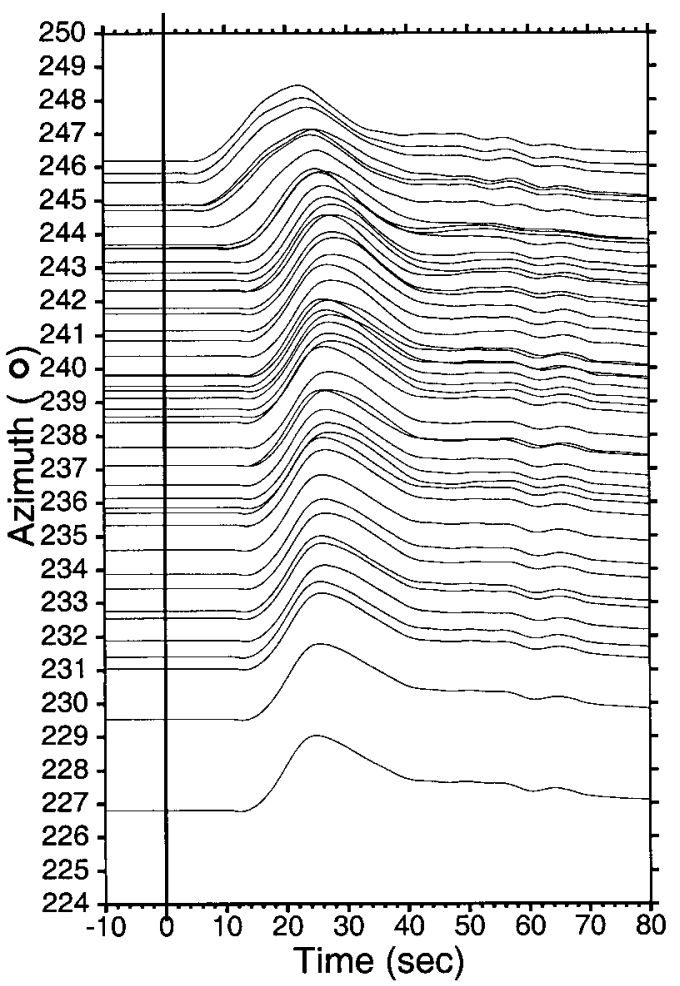

\section{SEM}

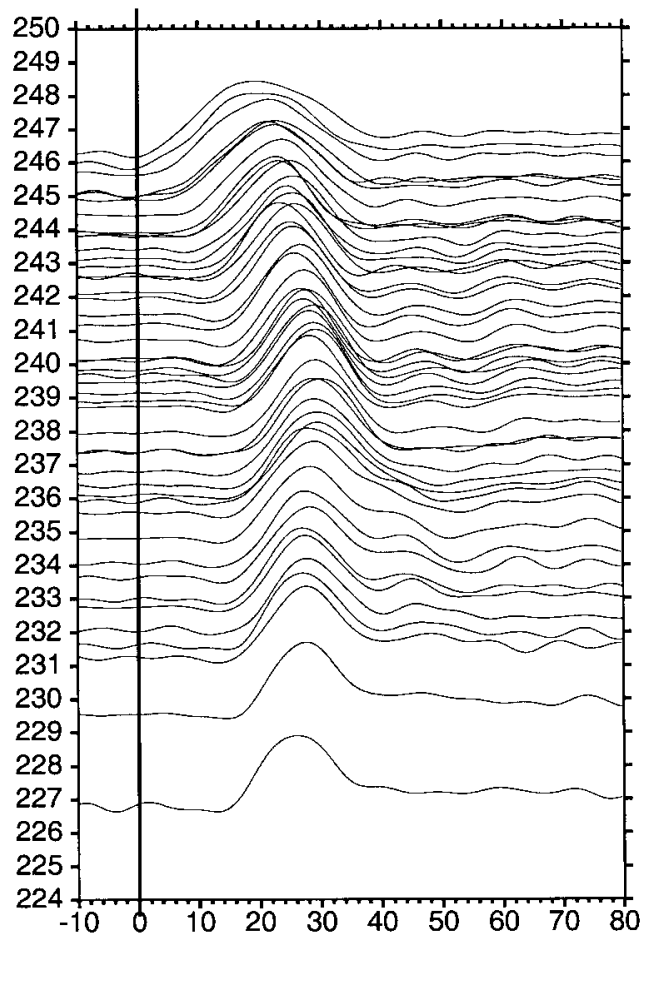

Figure 10. Comparison of synthetics generated with the DWKM code (analytical) against those from SEM (numerical). The amplitudes are all normalized to the SEM synthetic at the azimuth of $230^{\circ}$. Note the reduction in amplitude where the phases become multipathed or broadened at the edge position.

$$
O_{\mathrm{L}}(t) \equiv \frac{d}{d t}\left[\left(\frac{H(t)}{\sqrt{t}}-\frac{H\left(t-t_{\mathrm{f}}\right)}{\sqrt{t+t_{\mathrm{f}}}}\right) * \dot{D}(t)\right]
$$

for the lit region, and

$$
O_{\mathrm{D}}(t) \equiv \frac{d}{d t}\left[\left(\frac{H\left(t-t_{\mathrm{f}}\right)}{\sqrt{t}} * \dot{D}(t)\right]\right.
$$

for the diffracted zone. Note that the latter is a long-period correction because the singularity has been removed. An example set of these operators is given in Figure 9. Note that $O_{\mathrm{D}}(t)$ starts later because such diffracted arrivals travel further to get to the true receiver. The synthetics in the second and third columns in Figure 7 were generated in this manner. The longer the source duration, the greater the window sampled by the operators as apparent from examining this figure. Note that in a true diffraction application, we would compute the times $t_{1}$ and $t_{\mathrm{d}}$ at the reference arc. But because the ray paths travel nearly parallel from this position to the surface, we neglect their differential paths above the reference line.

A comparison of these DWKM synthetics against a 3D numerical code is given in Figure 10. The numerical code is called SEM which stands for the spectral-element method
(Komatitsch and Tromp, 1999, 2002). The 3D model is discussed in Ni et al. (2005) and contains sharp vertical walls. It proves difficult to exactly match the waveforms produced by the two methods because both contain a series of filters describing attenuation, source description, etc. Because we are interested in waveform changes that occur across the array, we generally pick a particularly simple observation and use it as an effective source or empirical source. In Figure 10 , we adopted the record near $230^{\circ}$ azimuth (SEM) for normalization. The DWKM synthetics are the same as in Figure 7 (third column) except for the change in this empirical source.

Two important features of the data are captured by these predictions. First, the travel-time delays of up to $10 \mathrm{~s}$ are quite apparent in the synthetics. However, they occur abruptly in the synthetics between $246^{\circ}$ and $244^{\circ}$, whereas in data, the transition is less sharp. Second, the double pulses near $246^{\circ}$ are reasonably well matched except the amplitude of the second arrival seems larger in the data. Both of these features argue for some of mismatch in geometry, that is, actual ray paths are not truly parallel to the structure. However, the most important point is in the interpretation of the second arrival. In 2D models or even 1D models, the only way to produce such a delayed pulse at these distances is by 


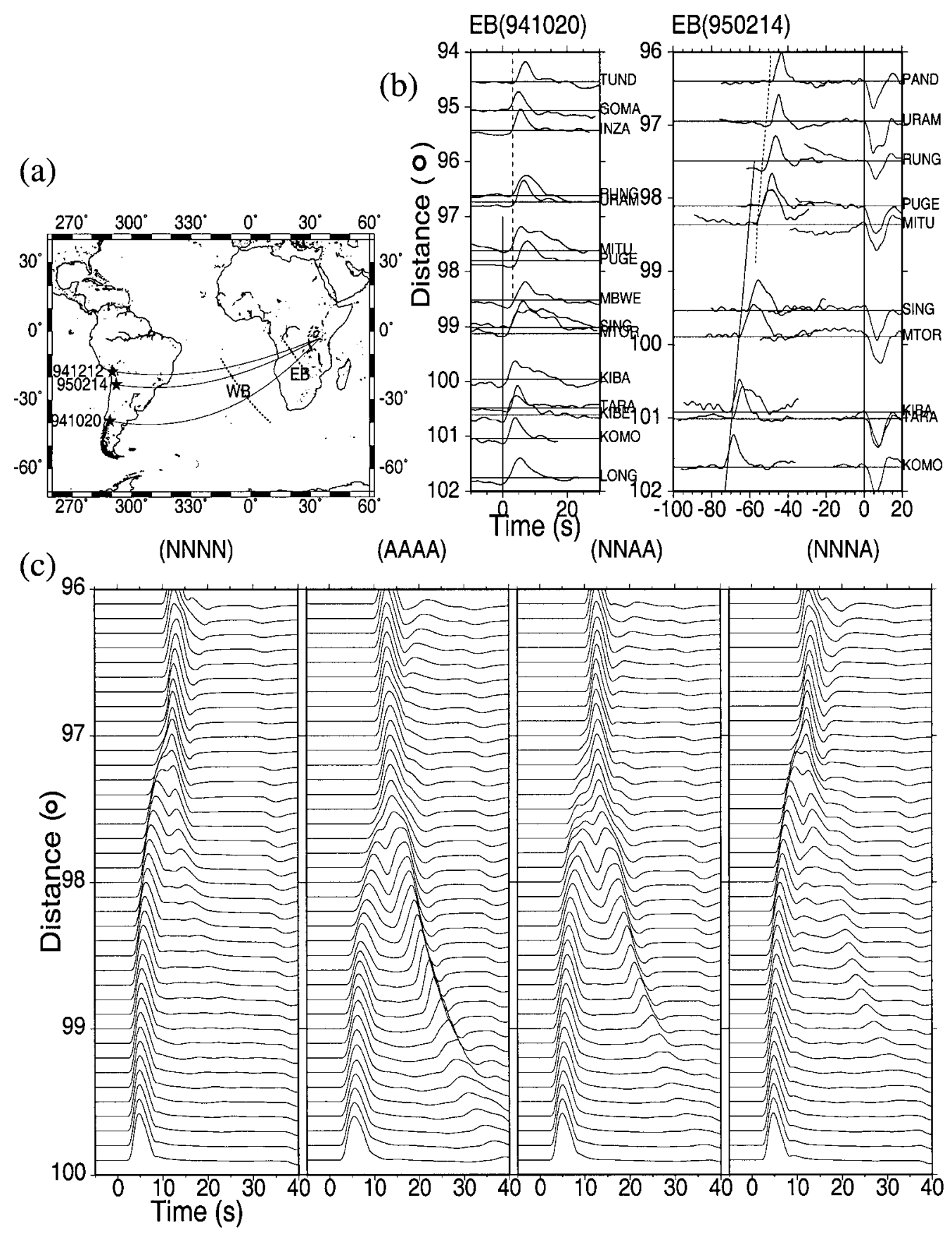

Figure 11. Boundary layer sensitivity testing for the 2D model presented in Figure 1. Great-circle paths are displayed in map view in panel (a) with some examples of observed SKS waveforms in panel (b). The arrivals are aligned on PREM for event 941020 and on observed $S H_{\mathrm{d}}$ for event 950214 . Note that the SKS is elongated relative to $S H_{\mathrm{d}}$ and shows a 5-s offset between $98^{\circ}$ and $101^{\circ}$. (c) Model $\mathrm{N}$ has a thermal boundary $60 \mathrm{~km}$ wide with a $1.5 \%$ velocity reduction. Model A has a $6 \%$ reduction instead, corresponding to a "plume-type layer." The 3D synthetics are computed by summing the weighted average of these $2 \mathrm{D}$ models. The two inside letters correspond to the "lit zone." 2D synthetics are generated for two simple cases in column one (NNNN) and column two (AAAA) for the phase SKS as it samples the eastern edge. The third column contains a 3D model where the model is $\mathrm{N}$ into the paper (north) and A out of the paper (south). The synthetics are simulated from four 2D sections, hence the four letters. The fourth column has only the more distant anomaly ( $200 \mathrm{~km}$ off the Great Circle). The column on the right contains observed waveforms from the Tanzania Array (data taken from Ni et al., 2002, displaying considerable variability). 
slowing down $S_{\mathrm{c}} S$ or by introducing a ULVZ (see, for example, Ni and Helmberger, 2001). Thus, sharp structures require special consideration in waveform modeling. It is difficult to locate complex boundary structure from paths traveling large distances along the edge. A more direct approach is examining the edge along perpendicular paths as introduced in Figure 1. Thus, we choose such a path setup for a second application, Figure 11. Data from three events recorded by the Tanzania Array (Fig. 11b) display complexity near the SKS crossover from slow (inside structure $<96^{\circ}$ ) to fast (outside $<102^{\circ}$ ) (Ni et al., 2002). The array has an extended cross alignment so that there is some mixing in azimuth and distance. Moreover, the edge is sampled at slightly different locations for each event (Fig. 11a). We again placed a horizontal reference plane directly above the 3D structure and added non-great-circle paths (in and out of the paper). Four strips containing velocity anomalies were placed in the two lit and differential zones for various combinations. Because the velocity contrast reaches $6 \%$ for the plume-style strip, the synthetics for the (AAAA) produce very strong diffractions. These data do not support such strong features, although elongated pulses are commonly observed as displayed and some datasets display such trailingoff features (Ni et al., 2005).

\section{Discussion and Conclusion}

Whereas global tomographic models display considerable variation, they tend to be quite smooth. Hence, the generation of 3D synthetics remains PREM-like (Ni et al., 2005). However, broadband observations from arrays are displaying considerable evidence for multipathing (i.e., Ni et al., 2005). To explain such data, one can suppose that tomographic models have been overly smoothed because of the present methodology of determining both the source location and structure simultaneously. To sharpen features, we can perform mapping procedures based on imposing functional rules (i.e., Sidorin et al., 1999). Because $S_{\text {cd }}$ is not observed globally, it is not included in such datasets nor is it produced by global models. It can be produced by adding sharpness as demonstrated in the previous study. Thus, new models can be created and easily tested against auxiliary waveform data by applying DWKM.

In the previous examples, the reference plane was inserted just above a particular strong structural feature, but a more general procedure would be to place it at the bottoming position or halfway between the source and receiver, as suggested in Burdick and Salvado (1986). The applicability of DWKM to such a problem needs to be established and will be pursued in future efforts.

In summary, we introduced a new approach in simulating 3D synthetics called the DWKM method. Instead of tracing rays through $3 \mathrm{D}$ structures, we treat the simpler problem of generating 2D synthetics (WKM) for azimuths near the receiver. The positions of these non-great-circle paths at virtual receivers are controlled by the source duration and the distance to the 3D target structure, essentially the Fresnel zone, $X=\sqrt{\sigma h T}$. We refer to the interior region as the "Lit" zone and exterior region as the "Diffracted" zone. The 2D WKM responses at these four virtual receivers are weighted and delayed relative to a square-root singularity centered on the true receiver. Results from these approximations are compared with the 3D numerical synthetics (SEM) with satisfactory results. Thus, we have developed a new tool for investigating $3 \mathrm{D}$ earth structure which is particularly useful for modeling broadband array data.

\section{Acknowledgments}

We thank Jeroen Tromp for running his SEM code for our model, and the reviewers. This work was supported by NSF Grant EAR-0229885. Contribution number 9037 of the Division of Geological and Planetary Sciences, California Institute of Technology.

\section{References}

Born, M., and E. Wolf (1964). Principles of Optics, The MacMillan Company, New York.

Burdick, L. J., and J. A. Orcutt (1979). A comparison of the generalized ray and reflectivity methods of waveform synthesis, Geophys. J. R. Astr. Soc. 58, 261-278.

Burdick, L. J., and C. A. Salvado (1986). Modeling body wave amplitude fluctuations using the three-dimension slowness method, J. Geophys. Res. 91, 12,482-12,496.

Červeny, V., and J. E. P. Soares (1992). Fresnel volume ray tracing, Geophysics 57, no. 7, 902-915.

Chapman, C. H. (1976). Exact and approximate generalized ray theory in vertically inhomogeneous media, Geophys. J. R. Astr. Soc. 46, 201233.

Chapman, C. H., and J. A. Orcutt (1985). The computation of body wave synthetic seismograms in laterally homogeneous media, Rev. Geophys. 23, 105-163.

Frazer, L. N., and M. K. Sen (1985). Kirchhoff-Helmholtz reflection seismograms in a laterally inhomogeneous multi-layered elastic medium. I. Theory, Geophys. J. R. Astr. Soc. 80, 121-147.

Gilbert, F., and D. V. Helmberger (1972), Generalized ray theory for a layered sphere, Geophys. J. R. Astr. Soc. 27, 57-80.

Gurnis, M., J. X. Metrovica, J. S. Ritsema, and H. van Heijst (2000). Constraining mantle density structure using geological evidence of surface uplift rates: the case of the African super plume, Geochem. Geophys. Geosyst. 1, 35.

Hager, B. H., R. W. Clayton, M. A. Richards, R. P. Comer, and A. M. Dziewonski (1985). Lower mantle heterogeneity; dynamic topography and the geoid, Nature 313, 541-545.

Helmberger, D. V. (1973). Numerical seismograms of long-period body waves, from seventeen to forty degrees, Bull. Seism. Soc. Am. 63, 633-646.

Helmberger, D. V. (1983). Theory and application of synthetic seismograms, in Earthquakes: Observation, Theory, and Interpretation, $\mathrm{H}$. Kanamori and E. Boschi (Editors), North-Holland, Amsterdam, vol. 37, 174-217.

Helmberger, D. V., and S. D. Malone (1975). Modeling local earthquakes as shear dislocations in a layered half space, J. Geophys. Res. 80, no. 35,4881 .

Helmberger, D. V., and J. E. Vidale (1988). Modeling strong motions produced by earthquakes with 2-D numerical codes, Bull. Seism. Soc. Am. 78, no. 1, 109-121.

Hilterman, F. J. (1975). Amplitudes of seismic waves: a quick look, Geophysics 40, 745-762. 
Ishii, M., and J. Tromp (1999). Normal-mode and free-air gravity constraints on lateral variations in velocity and density of Earth's mantle, Science 285, 1231-1236.

Komatitsch, D., and J. Tromp (1999). Introduction to the spectral-element method for 3D seismic wave propagation, Geophys. J. Int. 139, 806822 .

Komatitsch, D., and J. Tromp (2002). Spectral-element simulations of global seismic wave propagation. Part II: 3D models, oceans, rotation, and gravity, Geophys. J. Int. 150, 303-318.

Masters, G., G. Laske, H. Bolton, and A. Dziewonski (2000). The relative behavior of shear velocity, bulk sound speed, and compression velocity in the mantle, in Earth's Deep Interior, S. E. A. Karato (Editor), American Geophysical Monograph 117, 63-87.

Ni, S., and D. V. Helmberger (2001). Horizontal transition from fast to slow structures at the core-mantle boundary; South Atlantic, Earth Planet. Sci. Lett. 187, 301-310.

Ni, S., and D. V. Helmberger (2003a). Ridge-like lower mantle structure beneath South Africa, J. Geophys. Res. 108, no. B2, 2094.

Ni, S., and D. V. Helmberger (2003b). Seismological constraints on the South African superplume; could be the oldest distinct structure on Earth, Earth Planet. Sci. Lett. 206, 119-131.

Ni, S., X. Ding, and D. V. Helmberger (2000). Constructing synthetics from deep earth tomographic models, Geophys. J. Int. 140, 71-82.

Ni, S., D. V. Helmberger, and J. Tromp (2005). 3D structure of the African Superplume from waveform modeling, Geophys. J. Int. 160, 1-12.

Ni, S., E. Tan, M. Gurnis, and D. V. Helmberger (2002). Sharp sides to the African super plume, Science 296, 1850-1852.

Scott, P. (1985). Applications of the Kirchhoff-Helmholtz integral to problems in body wave seismology, Ph.D. Thesis, California Institute of Technology, Pasadena.
Scott, P., and D. V. Helmberger (1985). Applications of the transmitted Kirchhoff-Holmholtz method to transmitted body waves and possible structural effects at NTS, Bull. Seism. Soc. Am. 75, no. 1, 131-156.

Sidorin, I., M. Gurnis, and D. V. Helmberger (1999). Evidence for a ubiquitous seismic discontinuity at the base of the mantle, Science 286, 1326-1331.

Stead, R. J., and D. V. Helmberger (1988). Numerical-analytical Interfacing in two dimensions with applications to modeling NTS seismograms, Pure Appl. Geophys. 128, 153-174.

Tackley, P. (2000). Mantle convection and plate tectonics: toward an integrated physical and chemical theory, Science 288, 2002-2007.

Wen, L. (2001). Seismic evidence for a rapidly varying compositional anomaly at the base of the Earth's mantle beneath the Indian Ocean, Earth Planet. Sci. Lett. 194, 83-95.

Wen, L., and D. V. Helmberger (1998). A two-dimensional P-SV hybrid method and its application to modeling localized structures near the core-mantle boundary, J. Geophys. Res. 103, no. B8, 17,901-17,918.

Williams, Q., J. S. Revanaugh, and E. J. Garnero (1998). A correlation between ultra-low basal velocities in the mantle and hot spots, Science 281, 546-549.

Zhu, L., and D. V. Helmberger (1998). Moho Offset Across the northern margin of the Tibetan Plateau, Science 281, 1170-1172.

Seismological Laboratory 252-21

California Institute of Technology

Pasadena, California 91125

Manuscript received 12 January 2004. 